\title{
Millimetre observations of infrared carbon stars ${ }^{\star \star \star}$
}

\section{The data}

\author{
M. A. T. Groenewegen ${ }^{1}$, M. Sevenster ${ }^{2}$, H. W. W. Spoon ${ }^{3}$, and I. Pérez ${ }^{2,4}$ \\ ${ }^{1}$ Instituut voor Sterrenkunde, PACS-ICC, Celestijnenlaan 200B, 3001 Heverlee, Belgium \\ 2 Mount Stromlo and Siding Spring Observatory, Cotter road, Weston ACT, Australia \\ 3 Kapteyn Astronomical Institute, Postbus 800, 9700 AV, Groningen, The Netherlands \\ ${ }^{4}$ Departamento de Astronomía, Universidad de Chile, Casilla 36-D, Santiago, Chile
}

Received 1 March 2002 / Accepted 15 May 2002

\begin{abstract}
Millimetre observations of IRAS selected red carbon stars are presented. About 260 stars have been observed with SEST and IRAM in the CO (1-0) and CO (2-1) lines and partially in $\mathrm{HCN}(1-0)$ and $\mathrm{SiO}(3-2)$. An overall detection rate, in at least one line, of about $80 \%$ is achieved. The survey represents the second largest survey for AGB stars, and the largest ever for carbon stars. Two new detections in $\mathrm{SiO}(3-2)$ in carbon stars are reported. When available, the $\mathrm{SiO} / \mathrm{HCN}$ and $\mathrm{HCN} / \mathrm{CO}(1-0)$ line ratios are consistent with the ratios expected for carbon stars.
\end{abstract}

Key words. circumstellar matter - stars: mass-loss - stars: AGB and post-AGB

\section{Introduction}

The orbits of stars, moving in the global potential of the Galaxy, are functions of the integrals of motion in that potential only. For an axisymmetric potential, under the assumption that the radial-velocity dispersion equals the vertical-velocity dispersion, these integrals are the binding energy $E$ and the vertical angular momentum $L_{z}$. The dynamical behaviour of a particular sample of stars is then fully characterised by a distribution function in these two variables (see Sevenster et al. 1995, and references therein). This distribution function is found by comparing the moments of the observed distribution to the projected moments of model distributions on a grid in galactic coordinates $l, b$. The noise in the input moments is, naturally, dependent of the number of stars observed, and typically a few hundred stars are necessary to constrain models on an extended region of the sky. The comparison is performed with a quadratic-programming method (DeJonghe 1989). The spatial

Send offprint requests to: M. Groenewegen,

e-mail: groen@ster.kuleuven.ac.be

* Based on observations collected at the European Southern Observatory, La Silla, Chile within program ESO 60.E-0103, 62.L0128, 64.L-0012 and 66.D-0027. Also based on observations with the IRAM telescope, Granada, Spain under programs 98-97, 141-97 and 010-99

$\star \star$ The complete Fig. 1 is only available in electronic form at http://www.edpsciences.org. The complete Table 3 is only available in electronic form at the CDS via anonymous ftp to cdsarc.u-strasbg.fr $(130.79 .128 .5)$ or via http://cdsweb.u-strasbg.fr/cgi-bin/qcat?J/A+A/390/501 distribution and the kinematics of a certain population of stars will in general not be self-consistent, since the velocities are determined by the global potential rather than the potential derived from the spatial distribution via Poisson's equation. This is fully taken into account by the modelling method. The resulting analytic distribution functions can very easily be used for further analysis. For Galaxy studies, the technique has been successfully applied to Planetary Nebulae (Durand et al. 1996) and OH/IR stars (Sevenster et al. 1995).

Some time ago, we started a study into the dynamical behaviour of carbon stars. The aim of this project is to compare the dynamical behaviour of carbon stars to OH/IR stars and to test the hypothesis that the so-called "infra-red" carbon stars form a more massive population than the traditional well-known "optical" carbon stars (see e.g. Groenewegen et al. 1995). To investigate this, we acquired two samples. One sample of 700 optical carbon stars, for which radial velocities are available, was taken from Aaronson et al. (1989, 1990). The analysis was done on a smaller sub-sample of 500 that was selected to make the sample more homogeneous. First results are presented in Sevenster et al. (2000). As expected for such relatively low-mass AGB stars the scale height is large (650 vs. $200 \mathrm{pc}$ for low-latitude OH/IR stars). There is no discontinuity between their dynamical distribution and that of the lowmass $\mathrm{OH} / \mathrm{IR}$ stars, showing that, despite the very different radial ranges occupied by $\mathrm{O}$-rich and $\mathrm{C}$-rich stars, these do form one dynamical (AGB) population.

To ascertain that this applies to all AGB stars, a second sample of infrared carbon stars, to be compared to the more 
massive $\mathrm{OH} / \mathrm{IR}$ stars, was selected from the literature, as discussed below. Since only part of those have known radial velocities, an observational program was initiated to obtain radial velocities for the other stars. As these stars, almost by definition, have weak or no optical counterparts and thick circumstellar shells, the most efficient way to obtain the radial velocity is through millimetre line spectroscopy.

The main aim of the paper is therefore to provide the radial velocities of a large sample of infrared carbon stars as input to further analysis of the dynamical properties of carbon stars. However, as a by-product, the expansion velocities and line strengths are also obtained of the stars. The sample is introduced in Sect. 2, and the observations are presented in Sect. 3. The results are briefly discussed in Sect. 4.

The new observations are combined with mm-data available in the literature and analysed in terms of the spatial dependence of the expansion velocity, dust and gas mass loss rate and replenishment of the interstellar medium in the accompanying paper (Groenewegen et al. 2002). The analysis of distribution functions and the comparison to the dynamical properties of other populations of evolved stars will be reported in a third paper (Sevenster et al. 2002, in preparation).

\section{The sample}

The sample of (very probable) infrared carbon stars was selected as follows. In a first step, objects that fulfilled the following criteria were selected from the IRAS Point Source Catalog version 2 (PSC): Flux quality 3 at 12 and $25 \mu \mathrm{m}$, and $\geq 2$ at $60 \mu \mathrm{m}$, flux ratios $S_{12} / S_{25}<3, S_{25} / S_{60}>2.75$, and finally $S_{12}>20$ Jy or IRAS LRS classification equal to $4 \mathrm{n}$. The former flux-ratio is used to select stars with an far-IR excess, while the latter flux-ratio assures that red OH/IR stars and Planetary Nebulae are excluded. Applying these criteria resulted in 2088 stars which contains M- and S-stars as well.

In the next step, the following stars were removed: those which are associated in the PSC with the catalogue of S-stars, and stars with a spectral type $\mathrm{M}$ in the Michigan or SAO catalogue (as listed in the PSC).

In the next step, the IRAS LRS spectra (from the LRS catalogue, Volk \& Cohen 1989, Volk et al. 1991) and the SIMBAD database were checked for the remaining stars. A detection in a $\mathrm{SiO}$ or $\mathrm{OH}$ thermal or maser line implied that the star would not be observed by us. The visual inspection of the LRS spectra ensured that stars with silicate emission, but nevertheless classified as $4 \mathrm{n}$, were removed.

There are known infrared carbon stars which are not classified as LRS $=4 \mathrm{n}$, namely the extremely red carbon stars (Group v in Groenewegen et al. 1992; also see Volk et al. 1992). From these papers we selected those stars that fullfilled the same criteria on flux-quality and flux ratios as above.

The total sample contains 379 stars. For those we searched in the literature (up to January 1997 when this program was originally initiated) for available radial velocities from $\mathrm{CO}$ line emission. Hundred twenty-four stars have a reliable radial velocity reported (from the Loup et al. 1993 catalogue, Kastner et al. 1993 and Groenewegen et al. 1996; also see
Neri et al. 1998, Knapp et al. 1998, Josselin et al. 1998 for improved observations for a few of these stars). The remaining 255 were put on the observing list and here we report on millimetre observations of these stars.

\section{The observations}

Observations were performed with IRAM on 3 different occasions and with the SEST on 4 different occasions. The stars that were observed during the different runs are listed in Table 1; some stars were observed more than one time. Table 1 also lists the coordinates used in the observations which have been taken from the IRAS database. The accuracy of these coordinates is of order 10-15" which is comparable to the smallest beam size used here (CO 2-1 beam at IRAM). However the detection rate at IRAM is $90 \%$, and of the non-detections 5\% are likely inhibited by interstellar contamination so that non-detections due to incorrect input coordinates are a minor issue.

The IRAM data were taken between 18 and 22 October 1997 (observers MG and HS), 22 and 28 April 1998 (observer MG) and between 23 and 27 July 1999 (observers MG and MS). In October 1997 the ${ }^{12} \mathrm{CO}(2-1)$, HCN (1-0) and $\mathrm{SiO}(3-2)$ lines were observed simultaneously using the $1.3 \mathrm{~mm}, 2 \mathrm{~mm}$ and $3 \mathrm{~mm}$ SIS receivers. Detection of the $\mathrm{HCN}$ or $\mathrm{SiO}$ line could confirm or deny the carbon star character. Both the two $1 \mathrm{MHz}$ filter banks (for $\mathrm{CO}$ and $\mathrm{SiO}$ ) and the auto correlator (for $\mathrm{HCN}$, set to a channel spacing of $320 \mathrm{KHz}$ ) were used as back-ends.

In April 1998 the same configuration of front-ends, backends and observed lines was used for the first four observing slots. For the final two slots both $3 \mathrm{~mm}$ receivers were tuned to the ${ }^{12} \mathrm{CO}(1-0)$ lines, and both $1.3 \mathrm{~mm}$ receivers were tuned to the ${ }^{12} \mathrm{CO}(2-1)$ line. The $1 \mathrm{MHz}$ back-end was split and connected to the $1.3 \mathrm{~mm}$ receivers, and the auto correlator was split and connected to the $3 \mathrm{~mm}$ receivers (channel spacing $320 \mathrm{kHz}$ ). The brightest sources had preferentially been observed first and based on the results, it seemed unlikely to detect $\mathrm{HCN}$ in the weaker sources. On the other hand, with the new set-up a theoretical gain of $\sqrt{2}$ in signal-to-noise could be obtained on the CO lines, which turned out to be useful as many of our detections are quite faint.

In July $1999, \mathrm{HCN},{ }^{12} \mathrm{CO}(1-0)$ and ${ }^{12} \mathrm{CO}(2-1)$ were observed simultaneously. The $1 \mathrm{MHz}$ back-end was used on the $\mathrm{CO}(2-1)$ line, and the auto correlator was set to a channel spacing of $320 \mathrm{KHz}$ and used for $\mathrm{CO}(1-0)$ and $\mathrm{HCN}$.

On all occasions the targets were observed using wobbler switching with throws between 90 and $150^{\prime \prime}$ in azimuth; pointing and focus were checked every few hours.

The SEST data were taken between 10-12 and 14 March 1998 (observer MG), 9 and 14 March 1999 (observer MG), 23 and 28 October 1999 (observer MG), and between 25 and 31 March 2001 (observers MG and IP). On all occasions, ${ }^{12} \mathrm{CO}(1-0)$ and ${ }^{12} \mathrm{CO}(2-1)$ were observed simultaneously using the $1.3 \mathrm{~mm}$ and $3 \mathrm{~mm}$ SIS receivers. The dual beam switching mode was used with a throw of $2^{\prime} 26^{\prime \prime}$ in azimuth. One of two available acousto-optical spectrometers was split and connected to the two receivers. The channel separation is $0.7 \mathrm{MHz}$. Focus and pointing were checked every few hours, and almost 
Table 1. The stars observed.

\begin{tabular}{|c|c|c|c|}
\hline $\begin{array}{l}\text { IRAS } \\
\text { Name }\end{array}$ & $\begin{array}{r}\text { RA } \\
(1950)\end{array}$ & $\begin{array}{r}\text { Dec } \\
(1950)\end{array}$ & Observed \\
\hline $00422+5310$ & 04216.7 & 531024 & iram jul99 \\
\hline $01022+6542$ & 10212.6 & 654254 & iram apr98 \\
\hline $01080+5327$ & 10802.3 & 532738 & iram apr98 \\
\hline $01443+6417$ & 14419.6 & 641758 & iram apr98 \\
\hline $02345+5422$ & 23434.6 & 542219 & iram apr98 \\
\hline $02596+6639$ & 25937.5 & 663930 & iram jul99 \\
\hline $03157+3258$ & 31542.9 & 325840 & iram apr98; sest mar01 \\
\hline $03238+6034$ & 32352.3 & 603430 & iram apr98 \\
\hline $03277+5120$ & 32744.5 & 512009 & iram jul99 \\
\hline $03293+6038$ & 32921.6 & 603820 & iram apr98 \\
\hline $03385+5927$ & 33834.1 & 592730 & iram apr98 \\
\hline $03557+4404$ & 35542.1 & 440439 & iram apr98 \\
\hline $04127+5030$ & 41245.9 & 503009 & iram jul99 \\
\hline $04179+5951$ & 41755.6 & 595143 & iram apr98 \\
\hline $04297+2941$ & 42945.2 & 294149 & iram apr98; iram jul99; sest mar01 \\
\hline $04365+6349$ & 43631.3 & 634911 & iram oct 97 \\
\hline $04369+4501$ & 43655.0 & 450144 & iram oct97; iram jul99 \\
\hline $04449+4951$ & 44455.7 & 495153 & iram jul99 \\
\hline $05223+4704$ & 52223.6 & 470455 & iram oct 97 ; iram apr98 \\
\hline $05261+4626$ & 52609.1 & 462633 & iram oct 97 ; iram jul99 \\
\hline $05316+1757$ & 53140.1 & 175756 & iram jul99 \\
\hline $05428+1215$ & 54248.2 & 121506 & iram jul99 \\
\hline $05440+4311$ & 54401.7 & 431149 & iram jul99 \\
\hline $05447+1321$ & 54446.6 & 132136 & iram apr98; iram jul99; sest mar01 \\
\hline $06003+4747$ & 60021.7 & 474752 & iram oct $97 ;$ iram jul99 \\
\hline $06088+1909$ & 60850.9 & 190904 & iram jul99 \\
\hline $06181+0406$ & 61807.3 & 040636 & iram jul99 \\
\hline $06183+1135$ & 61819.3 & 113542 & iram jul99; sest oct99 \\
\hline $06192+0722$ & 61915.7 & 072230 & iram jul99; sest mar01 \\
\hline $06315+1606$ & 63130.9 & 160655 & iram jul99 \\
\hline $06323+3015$ & 63219.1 & 301514 & iram jul99; sest oct99 \\
\hline $06344-0124$ & 63429.3 & -012426 & iram jul99 \\
\hline $06348+3114$ & 63450.9 & 311423 & iram jul99; sest mar01 \\
\hline $06378-0527$ & 63751.3 & -052711 & sest mar98 \\
\hline $06447+0817$ & 64442.5 & 081718 & iram jul99 \\
\hline $06462-4157$ & 64616.7 & -415744 & sest mar99 \\
\hline $06471+0301$ & 64708.3 & 030143 & iram jul99 \\
\hline $06528-4218$ & 65252.2 & -421802 & sest mar98 \\
\hline $06558+2853$ & 65552.2 & 285302 & iram oct97; sest mar01 \\
\hline $06585-4111$ & 65834.4 & -411140 & sest oct99 \\
\hline $06588-2138$ & 65848.3 & -213846 & iram jul99; sest mar99; sest mar01 \\
\hline 07073-1944 & 70721.9 & -194445 & sest mar99 \\
\hline 07080-0106 & 70802.5 & -010627 & iram jul99 \\
\hline $07085-0018$ & 70833.7 & -001812 & sest oct99 \\
\hline 07149-0046 & 71459.5 & -004626 & sest oct99 \\
\hline 07161-0111 & 71606.2 & -011119 & iram jul99 \\
\hline $07170+0721$ & 71703.9 & 072132 & iram jul99 \\
\hline 07220-2324 & 72201.0 & -232450 & iram jul99; sest mar99; sest oct99; sest mar01 \\
\hline $07266-0541$ & 72641.2 & -054121 & sest mar99 \\
\hline 07336-1006 & 73340.2 & -100610 & sest mar98 \\
\hline $07356-3549$ & 73537.6 & -354920 & sest mar99; sest oct99 \\
\hline $07368-2833$ & 73650.3 & -283341 & sest mar98 \\
\hline $07373-4021$ & 73722.1 & -402149 & sest oct99 \\
\hline $07546-2551$ & 75437.1 & -255141 & iram ju199; sest oct99; sest mar01 \\
\hline 07553-0907 & 75527.3 & -090751 & iram jul99; sest mar01 \\
\hline 08086-3905 & 80839.3 & -390520 & sest mar98 \\
\hline
\end{tabular}


Table 1. continued.

\begin{tabular}{|c|c|c|c|}
\hline Name & $\begin{array}{r}\text { RA } \\
(1950) \\
\end{array}$ & $\begin{array}{r}\text { Dec } \\
(1950) \\
\end{array}$ & Observed \\
\hline $08119-3627$ & 81155.7 & -362747 & sest oct99 \\
\hline $08129-1236$ & 81258.0 & -123607 & iram jul99; sest mar99; sest oct99 \\
\hline $08250-2605$ & 82505.8 & -260538 & sest oct99 \\
\hline $08276-5125$ & 82741.9 & -512509 & sest mar98 \\
\hline $08292-3828$ & 82914.4 & -382801 & sest oct $99 ;$ sest mar01 \\
\hline 08304-4313 & 83027.7 & -431329 & sest mar98; sest mar99; sest mar01 \\
\hline 08305-3314 & 83033.9 & -331458 & sest mar98 \\
\hline 08340-3357 & 83404.3 & -335708 & sest mar99 \\
\hline $08353-3424$ & 83523.3 & -342411 & sest oct99 \\
\hline $08416-2525$ & 84140.4 & -252548 & sest mar98 \\
\hline 08439-2734 & 84358.1 & -273447 & sest oct99 \\
\hline 08470-5710 & 84705.6 & -571020 & sest oct99 \\
\hline 08534-5055 & 85327.2 & -505547 & sest mar99; sest oct99 \\
\hline $08535-4724$ & 85330.4 & -472426 & sest oct99; sest mar01 \\
\hline $08544-4431$ & 85429.5 & -443144 & sest oct $99 ;$ sest mar01 \\
\hline $08556-5717$ & 85541.3 & -571709 & sest oct99 \\
\hline 09164-5349 & 91627.6 & -534944 & sest oct $99 ;$ sest mar01 \\
\hline 09176-5147 & 91738.5 & -514742 & sest oct99 \\
\hline $09178-5556$ & 91751.9 & -555613 & sest mar01 \\
\hline 09238-5309 & 92349.5 & -530938 & sest oct99; sest mar01 \\
\hline 09317-5116 & 93143.0 & -511653 & sest mar99 \\
\hline $09428-4341$ & 94250.8 & -434151 & sest oct99; sest mar01 \\
\hline 09450-4716 & 94502.0 & -471644 & sest mar99 \\
\hline 09485-4232 & 94835.2 & -423248 & sest oct99; sest mar01 \\
\hline 09496-5050 & 94938.1 & -505012 & sest mar98; sest oct99 \\
\hline $09533-6021$ & 95320.3 & -602110 & sest oct99 \\
\hline 09547-5522 & 95447.7 & -552254 & sest oct $99 ;$ sest mar01 \\
\hline 09587-5056 & 95847.6 & -505659 & sest mar01 \\
\hline $10002-4641$ & 100013.0 & -464147 & sest mar01 \\
\hline $10068-6341$ & 100649.0 & -634120 & sest mar01 \\
\hline $10098-5742$ & 100949.5 & -574255 & sest mar98; sest mar99 \\
\hline $10231-5823$ & 102308.4 & -582354 & sest oct99; sest mar01 \\
\hline $10249-2517$ & 102456.5 & -251738 & sest mar01 \\
\hline $10375-4802$ & 103733.2 & -480210 & sest oct99 \\
\hline $10558-6537$ & 105549.3 & -653719 & sest oct $99 ;$ sest mar01 \\
\hline $11073-6325$ & 110719.2 & -632507 & sest oct99 \\
\hline $11079-6211$ & 110758.3 & -621133 & sest oct99; sest mar01 \\
\hline $11186-5528$ & 111837.4 & -552809 & sest mar98 \\
\hline $11272-6901$ & 112716.9 & -690138 & sest mar01 \\
\hline $11463-6320$ & 114622.0 & -632047 & sest mar98 \\
\hline $11514-5841$ & 115124.6 & -584144 & sest mar98 \\
\hline $12042-6355$ & 120413.0 & -635545 & sest mar99; sest oct99 \\
\hline $12142-6410$ & 121415.6 & -641029 & sest mar01 \\
\hline $12194-6007$ & 121926.2 & -600738 & sest mar99; sest oct99 \\
\hline $12195-6830$ & 121930.8 & -683045 & sest mar98; sest oct99 \\
\hline $12195-5527$ & 121935.4 & -552723 & sest mar99; sest mar01 \\
\hline $12216-6118$ & 122137.1 & -621812 & sest mar99 (observed at incorrect position); sest mar01 \\
\hline $12227-5045$ & 122242.3 & -504542 & sest mar99 \\
\hline $12298-5754$ & 122952.6 & -575457 & sest mar98 \\
\hline $12397-6447$ & 123947.5 & -644713 & sest mar98; sest oct99 \\
\hline $12419-6058$ & 124155.3 & -605840 & sest oct99 \\
\hline $12421-6217$ & 124208.4 & -621710 & sest mar01 \\
\hline $12464-6433$ & 124629.9 & -643339 & sest mar01 \\
\hline $12533-6118$ & 125323.4 & -611825 & sest mar01 \\
\hline $12550-7407$ & 125501.7 & -740730 & sest mar99 \\
\hline $12562-6003$ & 125613.3 & -600313 & sest mar01 \\
\hline $12569-6105$ & 125659.6 & -610509 & sest mar01 \\
\hline $12595-6035$ & 125931.9 & -603545 & sest mar98; sest mar01 \\
\hline
\end{tabular}


Table 1. continued.

\begin{tabular}{|c|c|c|c|}
\hline Name & $\begin{array}{r}\text { RA } \\
(1950) \\
\end{array}$ & $\begin{array}{r}\text { Dec } \\
(1950) \\
\end{array}$ & Observed \\
\hline $13031-5743$ & 130308.0 & -574318 & sest mar01 \\
\hline 13045-6404 & 130433.4 & -640421 & sest mar99 \\
\hline $13053-6341$ & 130518.2 & -634137 & sest mar98; sest mar99 \\
\hline 13064-6433 & 130625.5 & -643356 & sest mar99; sest oct $99 ;$ sest mar01 \\
\hline $13092-6026$ & 130916.3 & -602656 & sest mar01 \\
\hline $13208-6027$ & 132052.3 & -602749 & sest mar01 \\
\hline $13268-6226$ & 132651.7 & -622622 & sest mar98; sest mar99 \\
\hline $13343-5613$ & 133421.7 & -561321 & sest mar01 \\
\hline 13343-5807 & 133423.5 & -580755 & sest mar98; sest mar99 \\
\hline $13359-6014$ & 133554.6 & -601407 & sest mar01 \\
\hline $13482-6716$ & 134815.4 & -671608 & sest mar99 \\
\hline $13509-6348$ & 135056.8 & -634843 & sest mar99 \\
\hline $13595-5254$ & 135934.4 & -525422 & sest mar01 \\
\hline $14010-5927$ & 140101.7 & -592703 & sest oct99; sest mar01 \\
\hline $14122-5845$ & 141215.2 & -584523 & sest mar99 \\
\hline $14284-5245$ & 142825.5 & -524541 & sest mar99; sest mar01 \\
\hline $14309-5126$ & 143057.3 & -512617 & sest mar01 \\
\hline $14318-6107$ & 143152.5 & -610725 & sest oct99 \\
\hline $14358-6303$ & 143551.2 & -630308 & sest mar99 \\
\hline $14404-6320$ & 144025.1 & -632045 & sest oct99 \\
\hline $14443-5708$ & 144422.0 & -570809 & sest mar98 \\
\hline $14521-6058$ & 145206.1 & -605833 & sest mar01 \\
\hline $15043-5438$ & 150422.2 & -543825 & sest mar98; sest mar99 \\
\hline $15054-5458$ & 150526.9 & -545829 & sest mar99 \\
\hline $15202-5539$ & 152015.9 & -553905 & sest oct99 \\
\hline $15261-5702$ & 152606.3 & -570227 & sest mar98 \\
\hline $15330-5537$ & 153301.6 & -553730 & sest oct99 \\
\hline $15471-5644$ & 154706.3 & -564424 & sest mar98 \\
\hline $15488-4928$ & 154850.2 & -492827 & sest mar98 \\
\hline $16035-5330$ & 160333.6 & -533035 & sest mar99; sest mar01 \\
\hline $16047-5449$ & 160445.6 & -544927 & sest mar01 \\
\hline $16093-4808$ & 160918.8 & -480858 & sest oct99 \\
\hline $16123-4654$ & 161220.2 & -465454 & sest oct99 \\
\hline $16171-4759$ & 161709.5 & -475944 & sest mar98; sest oct99 \\
\hline $16265-5100$ & 162633.5 & -510059 & sest mar98; sest mar99; sest mar01 \\
\hline $16296-4417$ & 162942.0 & -441734 & sest oct $99 ;$ sest mar01 \\
\hline $16298-5349$ & 162952.2 & -534939 & sest oct99; sest mar01 \\
\hline 16304-3831 & 163029.3 & -383138 & sest oct99; sest mar01 \\
\hline $16469-4753$ & 164655.3 & -475353 & sest mar98; sest oct99 \\
\hline $16508-4620$ & 165049.0 & -462058 & sest oct99 \\
\hline $16545-4214$ & 165434.3 & -421450 & sest mar98; sest mar99 \\
\hline $16555-4456$ & 165530.5 & -445626 & sest mar01 \\
\hline $16562-5039$ & 165612.3 & -503930 & sest mar01 \\
\hline $17047-2848$ & 170446.4 & -284813 & sest mar98 \\
\hline $17050-4642$ & 170501.7 & -464223 & sest mar99 \\
\hline $17079-6554$ & 170759.4 & -655433 & sest mar98 \\
\hline $17103-3551$ & 171019.6 & -355153 & sest mar98; sest oct99 \\
\hline $17105-3746$ & 171035.8 & -374648 & sest oct99 \\
\hline 17130-3907 & 171304.8 & -390728 & sest mar99 \\
\hline $17155-4917$ & 171532.8 & -491732 & sest oct99 \\
\hline $17199-3512$ & 171959.5 & -351253 & sest mar01 \\
\hline $17209-3318$ & 172059.8 & -331837 & sest mar01 \\
\hline $17222-2328$ & 172215.8 & -232809 & iram oct $97 ;$ sest mar98 \\
\hline 17278-3937 & 172749.5 & -393733 & sest mar98; sest oct99; sest mar01 \\
\hline $17309-3412$ & 173054.8 & -341252 & sest mar99; sest oct99 \\
\hline $17375-3652$ & 173730.4 & -365210 & sest mar98 \\
\hline $17515-2407$ & 175133.2 & -240725 & iram oct97; sest mar98; sest mar01 \\
\hline
\end{tabular}


Table 1. continued.

\begin{tabular}{|c|c|c|c|}
\hline Name & $\begin{array}{r}\text { RA } \\
(1950)\end{array}$ & $\begin{array}{r}\text { Dec } \\
(1950)\end{array}$ & Observed \\
\hline $17547-3249$ & 175443.0 & -324908 & sest mar99 \\
\hline $17556+5813$ & 175537.4 & 581322 & iram apr98 \\
\hline $17599-4556$ & 175955.8 & -455645 & sest oct 99 ; sest mar01 \\
\hline 18030-1707 & 180304.6 & -170754 & iram jul99; sest mar01 \\
\hline $18038-1614$ & 180350.9 & -161400 & sest mar98; sest mar99 \\
\hline 18061-2739 & 180610.0 & -273913 & iram oct $97 ;$ sest mar99 \\
\hline $18082-2454$ & 180812.9 & -245429 & sest mar98 \\
\hline $18092-0437$ & 180917.5 & -043710 & iram oct 97 ; iram jul99 \\
\hline $18147-2215$ & 181442.0 & -221550 & sest mar98 \\
\hline $18230+0544$ & 182301.8 & 054418 & iram oct97 \\
\hline $18234-2206$ & 182327.7 & -220607 & sest mar98; sest oct99 \\
\hline $18244-0108$ & 182427.4 & -010803 & iram apr98; iram jul99 \\
\hline $18276-4717$ & 182737.7 & -471748 & sest mar98 \\
\hline $18289+0420$ & 182854.8 & 042037 & iram apr98; sest mar01 \\
\hline $18356-0951$ & 183540.3 & -095142 & iram oct $97 ;$ iram apr98; iram jul99 \\
\hline $19008+0726$ & 190053.1 & 072615 & iram oct $97 ;$ sest mar98 \\
\hline $19029+2017$ & 190257.4 & 201726 & iram oct 97 \\
\hline $19108+1155$ & 191053.1 & 115502 & iram oct97; iram apr98 \\
\hline $19136+6727$ & 191340.3 & 672708 & iram apr98; iram jul99 \\
\hline $19238+1159$ & 192353.6 & 115903 & iram apr98; iram jul99 \\
\hline $19248+0658$ & 192448.5 & 065803 & sest mar98 \\
\hline $19253+1918$ & 192522.0 & 191839 & iram apr98; iram jul99 \\
\hline $19276-0056$ & 192739.8 & -005631 & iram apr98; sest mar98 \\
\hline $19285+1808$ & 192835.7 & 180848 & iram apr98; iram jul99 \\
\hline $19289+1931$ & 192856.5 & 193149 & iram apr98; iram jul99 \\
\hline $19296+2227$ & 192937.7 & 222717 & iram apr98 \\
\hline $19304+2529$ & 193027.0 & 252941 & iram apr98; iram jul99 \\
\hline $19358+0917$ & 193549.0 & 091715 & iram apr98; iram jul99; sest mar98 \\
\hline $19381+3315$ & 193806.9 & 331523 & iram apr98; iram jul99 \\
\hline $19417+3053$ & 194143.4 & 305309 & iram apr98; iram jul99 \\
\hline $19419+3222$ & 194156.1 & 322211 & iram jul99 \\
\hline $19455+0920$ & 194532.4 & 092040 & iram apr98; iram jul99 \\
\hline $19455+2319$ & 194531.2 & 231906 & iram apr98; iram jul99 \\
\hline $19457+2346$ & 194542.5 & 234658 & iram apr98; iram jul99 \\
\hline $19485+3235$ & 194832.6 & 323552 & iram apr98 \\
\hline $19523+2414$ & 195221.9 & 241425 & iram apr98 \\
\hline $19524+2130$ & 195225.0 & 213037 & iram apr98 \\
\hline $19537+2212$ & 195347.5 & 221257 & iram apr98 \\
\hline $19552+3142$ & 195513.7 & 314217 & iram apr98; iram jul99 \\
\hline $19558+3333$ & 195553.3 & 333311 & iram apr98 \\
\hline $19559+3301$ & 195557.5 & 330126 & iram apr98; iram jul99 \\
\hline $20004+2943$ & 200029.1 & 294314 & iram jul99 \\
\hline $20014+2830$ & 200124.4 & 283010 & iram apr98 \\
\hline $20081+3228$ & 200812.7 & 322822 & iram oct97; iram apr98; iram jul99 \\
\hline $20084-1425$ & 200829.3 & -142505 & sest mar99; sest oct99; sest mar01 \\
\hline $20159+3134$ & 201558.9 & 313450 & iram jul99 \\
\hline $20171+3519$ & 201706.6 & 351921 & iram oct 97 ; iram apr98; iram jul99 \\
\hline $20200+3624$ & 202000.5 & 362400 & iram apr98; iram jul99 \\
\hline $20204+2914$ & 202029.1 & 291401 & iram apr98 \\
\hline $20253+3814$ & 202522.3 & 381448 & iram apr98; iram jul99 \\
\hline $20277+2958$ & 202742.3 & 295833 & iram apr98 \\
\hline $20282+3604$ & 202812.2 & 360431 & iram apr98; iram jul99 \\
\hline $20323+3153$ & 203222.9 & 315315 & iram apr98; iram jul99 \\
\hline $20331+4621$ & 203307.3 & 462116 & iram apr98; iram ju199 \\
\hline $20350+5954$ & 203504.0 & 595456 & iram jul99 \\
\hline $20351+2618$ & 203510.7 & 261846 & iram jul99; sest mar01 \\
\hline $20369+5131$ & 203657.4 & 513103 & iram apr98; iram jul99 \\
\hline $20461+4817$ & 204609.1 & 481749 & iram jul99 \\
\hline
\end{tabular}


Table 1. continued.

\begin{tabular}{lrrl}
\hline \hline Name & $\begin{array}{r}\text { RA } \\
(1950)\end{array}$ & $\begin{array}{r}\text { Dec } \\
(1950)\end{array}$ & Observed \\
\hline $20546+6405$ & 205438.1 & 640548 & iram jul99 \\
$20564+1857$ & 205629.2 & 185718 & iram jul99; sest oct99; sest mar01 \\
$20596+3833$ & 205936.4 & 383329 & iram apr98 \\
$21006+4720$ & 210040.1 & 472012 & iram apr98 \\
$21027+3704$ & 210242.1 & 370442 & iram apr98; iram jul99 \\
$21070+4711$ & 210700.8 & 471153 & iram apr98; iram jul99 \\
$21088+4546$ & 210851.7 & 454628 & iram apr98; iram jul99 \\
$21160+5546$ & 211605.9 & 554657 & iram jul99 \\
$21197-6956$ & 211946.9 & -695655 & sest mar98 \\
$21262+7000$ & 212615.1 & 700011 & iram jul99 \\
$21265+5042$ & 212630.7 & 504217 & iram jul99 \\
$21324+5537$ & 213224.7 & 553755 & iram apr98 \\
$21366+4529$ & 213637.9 & 452910 & iram apr98; iram jul99 \\
$21377+5042$ & 213746.9 & 504249 & iram apr98; iram jul99 \\
$21383+4513$ & 213818.5 & 451340 & iram apr98 \\
$21424+5821$ & 214224.7 & 582157 & iram apr98 \\
$21444+5053$ & 214426.8 & 505333 & iram apr98 \\
$22039+5328$ & 220357.9 & 532803 & iram jul99 \\
$22236+5002$ & 222339.8 & 500259 & iram jul99 \\
$23174+6810$ & 231729.9 & 681014 & iram apr98 \\
$23234+6434$ & 232329.2 & 643455 & iram apr98 \\
$23491+6243$ & 234909.1 & 624357 & iram apr98 \\
$23516+6430$ & 235141.8 & 643048 & iram apr98 \\
\hline
\end{tabular}

always found to be within twice the rms values of the last pointing model used by the telescope control software.

The data reduction consisted of several steps. First the individual measurements were reduced. Baselines were removed and all temperatures were put on a main-beam scale (the same applies for all temperatures presented in this paper). The conversion of observed antenna temperature into main-beam temperature requires knowledge of the forward and main beam efficiencies (listed in Table 2 together with the FWHM beam widths) and involves a comparison with calibration sources (e.g. Mauersberger et al. 1989 for IRAM). The comparison with the calibration sources (supplemented by consistency checks from stars observed more than once and from published results obtained with IRAM) indicate that the data are consistent. We estimate the final calibration for all observed lines to be accurate to $10 \%(1 \sigma)$. Then, the data of the same stars observed during different runs were co-added for IRAM and SEST separately.

The results are outlined in Table 3, available at the CDS, which lists the name of the object, the transition, the channel spacing, the rms noise, the peak temperature, the integrated intensity, the central velocity with respect to the local standard of rest and half the velocity width at zero intensity which equals the expansion velocity of the circumstellar shell. These determined quantities were determined either directly from the profiles or from fits to the profiles made with the reduction program cLAss. Typical uncertainties in the central velocity and the expansion velocity are $1 \mathrm{~km} \mathrm{~s}^{-1}$. The uncertainties in the peak temperature and the integrated intensity are dominated by the calibration uncertainties. Values flagged with a colon are uncertain. Upper limits are $3 \sigma$ values. The last column contains some
Table 2. The lines observed.

\begin{tabular}{llllll}
\hline \hline Line & $\begin{array}{l}\text { Frequency } \\
(\mathrm{MHz})\end{array}$ & Teles. & $F_{\text {eff }}$ & $B_{\text {eff }}$ & $\begin{array}{l}F W H M \\
\left({ }^{\prime \prime}\right)\end{array}$ \\
\hline${ }^{12} \mathrm{CO}(1-0)$ & 115271.204 & IRAM & 0.92 & 0.67 & 20.9 \\
& & SEST & & 0.70 & 45 \\
${ }^{12} \mathrm{CO}(2-1)$ & 230537.990 & IRAM & 0.86 & 0.39 & 10.4 \\
& & SEST & & 0.60 & 23 \\
$\mathrm{HCN}(1-0)$ & 88631.602 & IRAM & 0.92 & 0.76 & 27.0 \\
$\mathrm{SiO}(3-2, v=0)$ & 130268.702 & IRAM & 0.90 & 0.58 & 18 \\
\hline
\end{tabular}

Note. In July 1999 the beam efficiencies for IRAM were slightly better at $0.71(\mathrm{CO} 1-0), 0.48(\mathrm{CO} 2-1)$ and $0.77(\mathrm{HCN})$.

relevant remarks, for example when the spectrum was severely contaminated by interstellar contamination, or the system temperature when the weather was bad.

The calibrated profiles are shown in the Fig. 1, available in electronic form. Some of the strong interstellar spikes that appear on top of or near the stellar emission profiles and that may be several Kelvin strong have artificially been cut for display purposes. Some panels are intentionally left blank too facilitate comparison of different lines of the same object; the figures are ordered top to bottom, left to right.

\section{Results}

\subsection{General results}

The present survey is among the largest conducted for AGB stars in the CO lines and one with the highest detection rate. The largest single survey targeting IRAS objects (AGB stars, PNe, post-AGB, also some YSOs) is that by 
Table 3. The observed quantities.

\begin{tabular}{|c|c|c|c|c|c|c|c|c|}
\hline name & transition & $\begin{array}{c}\Delta v \\
\mathrm{~km} \mathrm{~s}^{-1}\end{array}$ & $\begin{array}{c}\mathrm{rms} \\
\mathrm{K}\end{array}$ & $\begin{array}{c}T_{\text {peak }} \\
\mathrm{K}\end{array}$ & $\underset{\mathrm{K} \mathrm{km} \mathrm{s}^{-1}}{\int T \mathrm{~d} v}$ & $\begin{array}{c}V_{\mathrm{LSR}} \\
\mathrm{km} \mathrm{s}^{-1}\end{array}$ & $\begin{array}{c}v_{\mathrm{e}} \\
\mathrm{km} \mathrm{s}^{-1}\end{array}$ & remarks \\
\hline \multirow[t]{3}{*}{$00422+5310$} & $2-1$ & 1.30 & 0.016 & 0.16 & 2.7 & 19.4 & 14.1 & \\
\hline & $1-0$ & 1.63 & 0.04 & 0.081 & 1.5 & 19.4 & 13.1 & \\
\hline & $\mathrm{HCN}$ & 1.06 & 0.011 & $<0.025$ & 0.4 : & & & \\
\hline \multirow[t]{3}{*}{$01022+6542$} & $2-1$ & 1.30 & 0.087 & & & & & $T_{\mathrm{sys}}=1400 \mathrm{~K}$ \\
\hline & $\mathrm{HCN}$ & 2.11 & 0.024 & & & & & \\
\hline & $\mathrm{SiO}$ & 2.30 & 0.022 & & & & & \\
\hline \multirow[t]{3}{*}{$01080+5327$} & $2-1$ & 1.30 & 0.048 & 0.47 & 15.0 & -20.0 & 22.6 & \\
\hline & $\mathrm{HCN}$ & 2.11 & 0.051 & $0.15:$ & 4.3 & & & \\
\hline & $\mathrm{SiO}$ & 2.60 & 0.024 & 0.05 : & 1.1: & & & \\
\hline \multirow[t]{4}{*}{$01443+6417$} & $2-1$ & 1.30 & 0.061 & 0.42 & 16.2 & -68.1 & 30.5 & \\
\hline & $1-0$ & 1.63 & 0.033 & 0.11: & $3.2:$ & $-70:$ & $28:$ & \\
\hline & $\mathrm{HCN}$ & 2.11 & 0.044 & 0.08 : & 3.4: & & & data corrupted blue wards of $-110 \mathrm{~km} \mathrm{~s}^{-1}$ \\
\hline & $\mathrm{SiO}$ & 2.30 & 0.022 & & 0.3: & & & \\
\hline \multirow[t]{3}{*}{$02345+5422$} & $2-1$ & 1.30 & 0.067 & 1.22 & 29.0 & -66.0 & 19.8 & \\
\hline & $\mathrm{HCN}$ & 2.11 & 0.070 & 0.25 & 5.3 & & & \\
\hline & $\mathrm{SiO}$ & 2.30 & 0.024 & & 0.3: & & & \\
\hline \multirow[t]{3}{*}{$02596+6639$} & $2-1$ & 1.30 & 0.019 & 0.15 & 2.7 & -41.8 & 15.8 & \\
\hline & $1-0$ & 0.81 & 0.024 & 0.048 & 0.9 & $-40:$ & & \\
\hline & $\mathrm{HCN}$ & 1.06 & 0.010 & & 0.2 : & & & \\
\hline \multirow[t]{5}{*}{$03157+3258$} & $2-1$ & 1.30 & 0.064 & & & & & $\operatorname{iram} ; T_{\text {sys }}=930 \mathrm{~K}$ \\
\hline & $\mathrm{HCN}$ & 1.06 & 0.092 & & & & & \\
\hline & $\mathrm{SiO}$ & 2.30 & 0.026 & & & & & \\
\hline & $2-1$ & 1.82 & 0.015 & 0.10 & 1.4 & -17.5 & 13.5 & sest \\
\hline & $1-0$ & 1.80 & 0.016 & 0.09: & 1.5 & -15.7 & 17.0: & \\
\hline \multirow[t]{3}{*}{$03238+6034$} & $2-1$ & 1.30 & 0.078 & 1.06 & 19.6 & -85.5 & 15.3 & \\
\hline & $\mathrm{HCN}$ & 2.11 & 0.073 & $<0.12$ & 1.2: & & & \\
\hline & $\mathrm{SiO}$ & 2.30 & 0.035 & $<0.10$ & 0.5 : & & & \\
\hline \multirow[t]{3}{*}{$03277+5120$} & $2-1$ & 1.30 & 0.031 & 0.11 & 2.3 & -30.2 & 17.9 & \\
\hline & $1-0$ & 0.81 & 0.047 & 0.11 & 2.6 & -28.9 & 16.3 & \\
\hline & $\mathrm{HCN}$ & 2.11 & 0.007 & 0.02 : & 0.3 : & & & \\
\hline \multirow[t]{3}{*}{$03293+6038$} & $2-1$ & 1.30 & 0.081 & 0.84 & 22.2 & -61.9 & 21.0 & \\
\hline & $\mathrm{HCN}$ & 2.11 & 0.068 & & 2.7: & & & \\
\hline & $\mathrm{SiO}$ & 2.30 & 0.038 & & 0.8: & & & \\
\hline \multirow[t]{4}{*}{$03385+5927$} & $2-1$ & 1.30 & 0.059 & 0.32 & 6.2 & -74.5 & 15.7 & \\
\hline & $1-0$ & 1.63 & 0.031 & 0.21 & 4.8 & -74.9 & 17.2 & \\
\hline & $\mathrm{HCN}$ & 2.11 & 0.063 & & $0.8:$ & & & \\
\hline & $\mathrm{SiO}$ & 2.30 & 0.021 & & & & & \\
\hline \multirow[t]{4}{*}{$03557+4404$} & $2-1$ & 1.30 & 0.039 & 0.36 & 6.0 & -49.2 & 14.7 & \\
\hline & $1-0$ & 1.63 & 0.031 & 0.39 & 6.8 & -47.6 & 15.0 & \\
\hline & $\mathrm{HCN}$ & 2.11 & 0.017 & 0.05 : & $0.8:$ & & & \\
\hline & $\mathrm{SiO}$ & 2.30 & 0.018 & & 0.2 : & & & \\
\hline \multirow{3}{*}{$04127+5030$} & $2-1$ & 1.30 & 0.022 & 0.23 & 6.5 & -0.1 & 17.9 & \\
\hline & $1-0$ & 1.63 & 0.016 & 0.066 & 1.4: & -0.2 : & 16.8: & \\
\hline & $\mathrm{HCN}$ & 2.11 & 0.008 & 0.048 & 1.1: & 1.3 & 17.1 & \\
\hline \multirow[t]{4}{*}{$04179+5951$} & $2-1$ & 1.30 & 0.055 & 0.65 & 20.3 & 4.2 & 23.4 & \\
\hline & $1-0$ & 1.63 & 0.033 & 0.28 & 9.4 & 4.4 & 22.5 & \\
\hline & $\mathrm{HCN}$ & 2.11 & 0.017 & 0.09 & 2.6 & 6.9 & 19.6 & \\
\hline & $\mathrm{SiO}$ & 2.30 & 0.024 & & 0.6: & & & \\
\hline \multirow[t]{5}{*}{$04297+2941$} & $2-1$ & 1.30 & 0.045 & $<0.12$ & 1.6: & & & $\operatorname{iram} ; T_{\text {sys }}=1450 \mathrm{~K}$ \\
\hline & $1-0$ & 1.63 & 0.018 & 0.11 & 2.5 & 3.5 & 17.7 & \\
\hline & $\mathrm{HCN}$ & 2.11 & 0.011 & 0.048 & 1.1 & 5.4 & 17.2 & \\
\hline & $2-1$ & 1.82 & 0.018 & & & & & sest; interstellar contamination \\
\hline & $1-0$ & 1.80 & 0.019 & 0.05: & & & & \\
\hline \multirow[t]{3}{*}{$04365+6349$} & $2-1$ & 1.30 & 0.124 & 0.43 & 7.1 & -47.0 & 14.2 & $T_{\text {sys }}=1870 \mathrm{~K}$ \\
\hline & $\mathrm{HCN}$ & 1.06 & 0.045 & 0.1 : & 1.7: & & & \\
\hline & $\mathrm{SiO}$ & 2.30 & 0.025 & & 0.5 : & & & \\
\hline
\end{tabular}



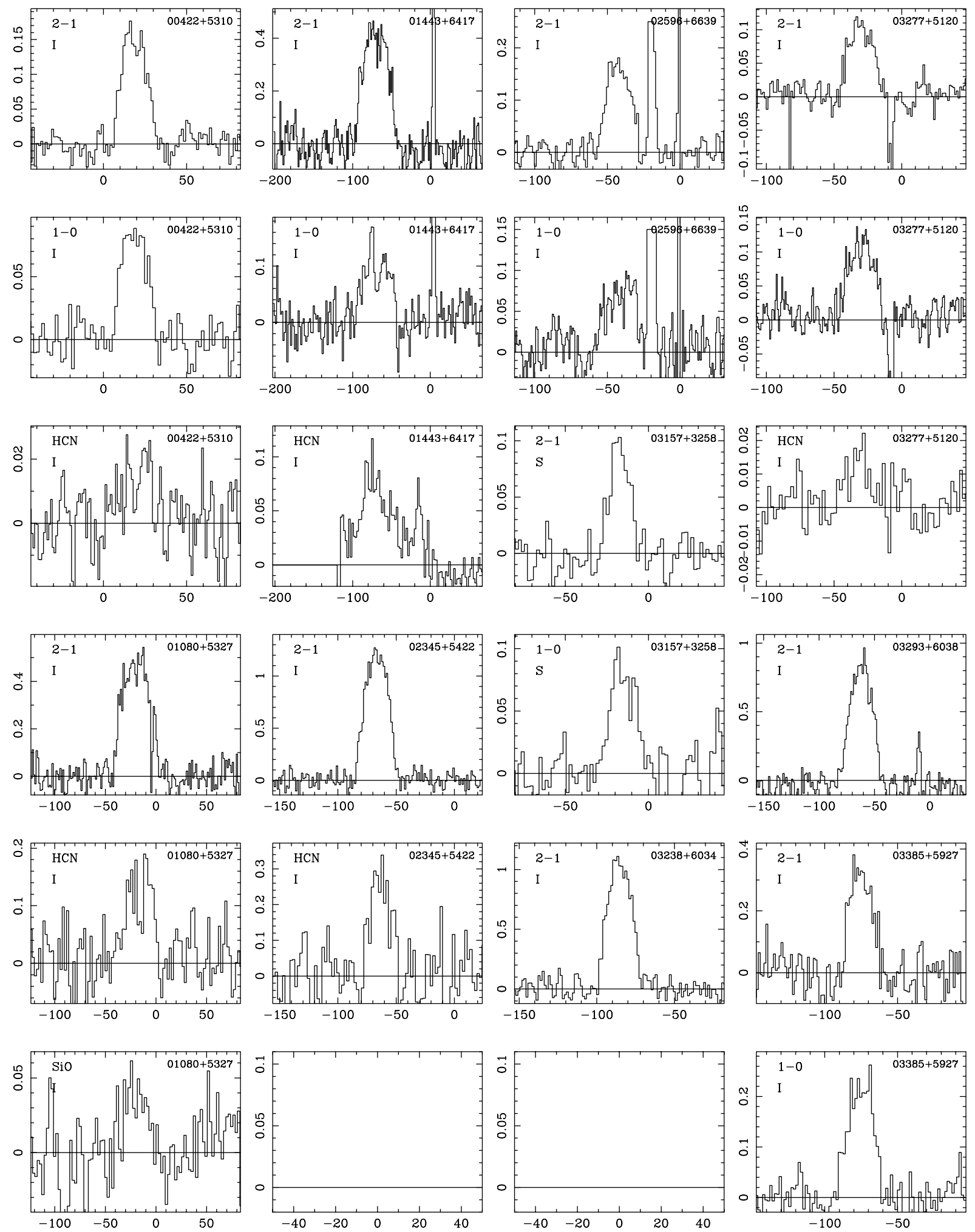

Fig. 1. Plotted are the velocity w.r.t. the LSR, and the main-beam temperature in Kelvin. The figures are ordered top to bottom, left to right. Some panels are intentially left blank in order to always have the observations of the same stars below each other so that the velocity scales are lined up for easy comparison.

Nyman et al. (1992), who observed 519 objects in the CO (1$0)$ line with SEST and detected 163 objects. Compared to their survey the present one uses more sensitive receivers and has the benefit of also observing $\mathrm{CO}(2-1)$ that is usually the stronger line. The present survey is probably the second largest aiming at AGB stars, and the largest specifically targeting carbon stars. A smaller survey, concentrating on carbon stars in the Galactic plane, is that by Kastner et al. (1993) who detected 47 out of 
58 stars observed in CO (1-0) and (2-1) with IRAM. Olofsson et al. (1993) detected 68 out of 99 observed bright $(K<2 \mathrm{mag})$ optically identified carbon stars with OSO, IRAM and SEST in CO (1-0) and (2-1). Kerschbaum et al. (1999) observed 109 Orich SR and Irregular variables in several CO lines with several telescopes, detecting 66. Numerous smaller surveys in CO, or mapping observations of previously $\mathrm{CO}$ detected objects, or surveys aimed at obtaining molecular abundances in previously $\mathrm{CO}$ detected objects exist.

In the end, all 252 stars on the observing list were observed. Of the 96 stars observed only with IRAM, 86 were detected in at least one line. Five of the non-detections may be inhibited by strong interstellar contamination. Of the 134 stars observed only with SEST, 101 were detected in at least one line, with 31 non-detections possibly inhibited by strong interstellar contamination. Twenty-one stars were observed with both telescopes, with 10 detected by IRAM and 13 by SEST in at least one line. Many of these sources observed with SEST had been observed with IRAM first (because of the expected higher sensitivity) under adverse weather conditions (the system temperatures have been listed in the 'remarks' column to mark these objects as candidates to be re-observed with IRAM under improved weather conditions). Overall $80 \%$ of the observed sources have been detected in at least one line. Many of the non-detections are sources in the Galactic plane observed with SEST which is less sensitive than IRAM to point sources and picks up more interstellar emission in its larger beam.

\subsection{Comparison of IRAM and SEST observations}

Since in almost all cases the SEST observations were only performed because the IRAM observations had been made under adverse weather conditions, or to obtain CO (1-0) data when the IRAM $1.3 \mathrm{~mm}$ receiver had been tuned to $\mathrm{HCN}$, only five stars have good data obtained with both telescopes. In these cases the ratio of the $\mathrm{CO}(1-0)$ and/or $\mathrm{CO}(2-1)$ integrated intensities between IRAM and SEST is between 3.2 and 4, what is approximately the expected ratio given the respective beam sizes.

\section{3. $\mathrm{SiO}$ and $\mathrm{HCN}$ data}

Of the 68 objects also observed in the $\mathrm{SiO}$ line, only one was convincingly detected (IRAS 19008+0726) and two marginally (IRAS $01080+5327,20596+3833$ ). These three are all optically identified carbon stars, being respectively number 4162 , 180, and 5089, in the General Catalog of Cool Carbon stars (GCCCS, Stephenson 1989). Their carbon star nature is beyond any doubt.

The presence of oxygen-rich molecules in the envelopes around carbon stars is well documented (Bujarrabal et al. 1994; Olofsson et al. 1989; Bieging et al. 2000 and references therein) and integrated intensities or line-ratios between two species are usually used to discriminate between $\mathrm{O}$ - and C-rich sources. Bujarrabal et al. (1994) quote a lower limit of 2.8 for Orich and an upper limit of 0.33 for C-rich sources for the $\mathrm{SiO}(3-2) / \mathrm{HCN}(1-0)$ ratio of the integrated intensities.
In the three stars just mentioned the ratio of the line intensities is $1.1 / 4.3=0.26,9.4 / 66.0=0.14$ and $1.1 / 1.5=0.73$ in IRAS $01080+5327,19008+0726,20596+3833$, respectively. In the last case, both $\mathrm{HCN}$ and $\mathrm{SiO}$ integrated intensities are uncertain, and for the first two cases the ratio is in agreement with the upper limit derived by Bujarrabal et al. (1994).

The ratio $\mathrm{HCN}(1-0) / \mathrm{CO}(1-0)$ is known to be as low as 0.17 in C-rich objects (Olofsson et al. 1998) and none of the twenty-four objects with reliable detections in both lines have an integrated intensity ratio lower than this.

In summary, the observations of the $\mathrm{SiO}$ and $\mathrm{HCN}$ lines give results that are in agreement with the line ratios expected for carbon stars.

Acknowledgements. This program was initiated, and part of the observations were obtained, when MG was a research fellow at the MaxPlanck Institut für Astrophysik, Garching, Germany. We would like to thank the program committees of ESO and IRAM for their continuing support. This research has made use of the SIMBAD database, operated at CDS, Strasbourg, France. René Oudmaijer is thanked for commenting upon an earlier version of the manuscript.

\section{References}

Aaronson, M., Blanco, V. M., Cook, K. H., \& Schechter, P. L. 1989, ApJS, 70, 637

Aaronson, M., Blanco, V. M., Cook, K. H., Olszewski, E. W., \& Schechter, P. L. 1990, ApJS, 73, 841

Bieging, J. H., Shaked, S., \& Gensheimer, P. D. 2000, ApJ, 543, 897

Bujarrabal, V., Fuente, A., \& Omont, A. 1994, ApJ, 421, L47

Dejonghe, H. 1989, ApJ, 343, 113

Durand, S., Dejonghe, H., \& Acker, A. 1996, A\&A, 310, 97

Groenewegen, M. A. T., de Jong, T., van der Bliek, N. S., Slijkhuis, S., \& Willems, F. J. 1992, A\&A, 253, 150

Groenewegen, M. A. T., Baas, F., de Jong, T., \& Loup, C. 1996, A\&A, 306,241

Groenewegen, M. A. T., Van den Hoek, L. B., \& de Jong, T. 1995, A\&A, 293, 381

Josselin, E., Loup, C., Omont, A., et al. 1998, A\&AS, 129, 45

Kastner, J. H., Forveille, T., Zuckerman, B., \& Omont, A. 1993, A\&A, 275,163

Kerschbaum, F., \& Olofsson, H. 1999, A\&AS, 122, 167

Knapp, G. R., Young, K., Lee, E., \& Jorissen, A. 1998, ApJS, 117, 209

Loup, C., Forveille, T., Omont, A., \& Paul, J. F. 1993, A\&AS, 99, 291

Mauersberger, R., Guélin, M., Martin-Pintado, J., et al. 1989, A\&AS, 79, 217

Neri, R., Kahane, C., Lucas, R., Bujarrabal, V., \& Loup, C. 1998, A\&AS, 130, 1

Nyman, L.-A., Booth, R. S., Carslström, U., et al. 1992, A\&AS, 93, 121

Olofsson, H., Eriksson, K., Gustafsson, B., \& Carlström, U. 1993, ApJS, 87, 267

Sevenster, M. N., DeJonghe, H., \& Habing, H. J. 1995, A\&A, 299, 689

Sevenster, M. N., Dejonghe, H., Van Caelenberg, K., \& Habing, H. J. 2000, A\&A, 355, 537

Stephenson, C. B. 1989, Publ. Warner \& Swasey Obs., 3

Volk, K., \& Cohen, M. 1989, AJ, 98, 931

Volk, K., Kwok, S., \& Langill, P. P. 1992, ApJ, 391, 285

Volk, K., Kwok, S., Stencel, R. E., \& Brugel, E. 1991, ApJS, 77, 607 